Rev. Int. Contam. Ambie. 37, 155-165, 2021

https://doi.org/10.20937/RICA.53782

\title{
EVALUACIÓN DEL CRECIMIENTO Y TOXIGENICIDAD DE Microcystis EN AGUAS ENRIQUECIDAS CON NUTRIENTES
}

\author{
Growth assessment and toxigenicity of Microcystis in nutrient-enriched waters \\ Aldo AQUINO-CRUZ*, Daniela Melissa TORRES CALDERÓN, \\ Erick Adolfo BARRIOS GARCÍA y Celia BULIT
}

Laboratorio de Plancton y Bioenergética, Departamento El Hombre y su Ambiente, Universidad Autónoma Metropolitana-Xochimilco, 04960 Ciudad de México, México.

*Autor para correspondencia: aldoaqcz@yahoo.com

(Recibido: octubre de 2019; aceptado: junio de 2020)

Palabras clave: aguas epicontinentales, nutrientes disueltos, cianotoxicidad, Ciudad de México.

\section{RESUMEN}

El crecimiento de diversas cianobacterias nocivas (Microcystis) se ha intensificado a nivel mundial como resultado de numerosas y rápidas alteraciones ambientales. En este estudio la cianobacteria Microcystis fue aislada de la Pista Olímpica de Remo y Canotaje Virgilio Uribe de la Ciudad de México y se investigaron: 1) la influencia del aumento de nitrógeno $(\mathrm{N})$ y fósforo $(\mathrm{P})$ en el crecimiento de Microcystis y 2) la toxigenicidad potencial de Microcystis mediante bioensayos de mortalidad de Artemia sp. En 2017, dos cepas clonales de Microcystis (Mc17C2 y Mc17C3) se establecieron en condiciones controladas de laboratorio. Dichas cepas crecieron en una cámara iluminada con irradianza de $19.6 \mu \mathrm{mol} / \mathrm{m}^{2} / \mathrm{s}$ con un fotoperiodo de $12 \mathrm{~h} \mathrm{luz:} 12 \mathrm{~h}$ oscuridad a temperatura ambiente $\left(\sim 24 \pm 2{ }^{\circ} \mathrm{C}\right)$. Se cultivó Artemia sp. y se seleccionaron juveniles del crustáceo para alimentarlos con diferentes concentraciones de Microcystis. El aumento en la concentración de $\mathrm{N}$ y $\mathrm{P}$ se asoció con mayores tasas de crecimiento de Microcystis; sin embargo, su crecimiento óptimo no coincidió con los niveles más altos de nutriente en los cultivos. La ingestión de Microcystis por juveniles de Artemia causó mortalidad del 50 al $100 \%$ en un lapso de 48-72 h cuando la abundancia fluctuó entre $3 \times 10^{5}$ y $3 \times 10^{6} \mathrm{cel} / \mathrm{mL}$. En general, Artemia sp. no mostró sensibilidad al consumo de Microcystis en las primeras $24 \mathrm{~h}$. Se concluye que el incremento y la disponibilidad de nutrientes disueltos conducen a mayores tasas de crecimiento de Microcystis con efecto potencial sobre el zooplancton.

Key words: epicontinental waters, dissolved nutrients, cyanotoxicity, Mexico City.

\begin{abstract}
The growth of several harmful cyanobacteria (Microcystis) has increased worldwide as a result of numerous and rapid environmental alterations. In this study, the toxigenic cyanobacterium Microcystis was isolated from a water body in Mexico City (Pista Olímpica de Remo y Canotaje Virgilio Uribe) with the aim to investigate: (1) the influence of increasing nitrogen $(\mathrm{N})$ and phosphorus $(\mathrm{P})$ on the growth of Microcystis and (2) the potential toxigenicity of Microcytis using mortality bioassays of Artemia sp.
\end{abstract}


In 2017, two clonal strains (Mc17C2 y Mc17C3) of Microcystis were established in monoculture under controlled laboratory conditions. Microcystis strains were grown in a culture chamber in vitro with irradiance $19.6 \mu \mathrm{mol} / \mathrm{m}^{2} / \mathrm{s}$ with a $12 \mathrm{~h}$ light: $12 \mathrm{~h}$ dark photoperiod, and at room temperature $\left(\sim 24 \pm 2^{\circ} \mathrm{C}\right)$. Artemia sp. was cultured and juvenile organisms were used and feed on different quantities of Microcystis strains. Increasing $\mathrm{N}$ and $\mathrm{P}$ concentrations lead to higher growth rates of Microcystis; however, its optimum growth rate did not match with the highest nutrient concentrations in the cultures. Microcystis ingestion by juvenile Artemia caused 50 to $100 \%$ of mortality in 48-72 h, when fed on abundance between $3 \times 10^{5}$ y $3 \times 10^{6}$ cells $/ \mathrm{ml}$. In general, Artemia was not susceptible to Microcystis ingestion within a time of $24 \mathrm{~h}$. In conclusion, higher availability of dissolved nutrients in the water leads to increasing growth rates of Microcystis which may cause potential effects on zooplankton.

\section{INTRODUCCIÓN}

Diversos sistemas acuáticos epicontinentales del territorio mexicano están siendo alterados negativamente debido a una compleja interacción de cambios y procesos ambientales, por ejemplo, la contaminación, la urbanización, la eutrofización, el cambio climático, alteraciones hidrológicas y los florecimientos algales nocivos (FAN) (Winder y Sommer 2012, Harke et al. 2016). Los FAN son fenómenos provocados por la abundancia y reproducción excesiva de microorganismos fotosintéticos, cianobacterias y algas de una o varias especies, en la columna de agua de ambientes acuáticos (GarcíaMendoza et al. 2016). En las últimas décadas, la frecuencia, permanencia, distribución geográfica e intensidad de los FAN se han incrementado en todo el mundo (Harke et al. 2016).

Distintas cianobacterias planctónicas formadoras de florecimientos (Microcystis, Planktothrix, Aphanizomenon, Nodularia, Cylindrospermopsis, Limnothrix) causan graves problemas ambientales, socioeconómicos y de salud reconocidos a nivel mundial (Paerl y Huisman 2008). Microcystis es uno de los géneros cianobacteriales dominantes en aguas epicontinentales (Harke et al. 2016), cuyos florecimientos pueden resultar perjudiciales debido, entre otras cuestiones, a la presencia de microcistinas, toxinas oxidantes generadoras de diferentes problemas de salud (O'Neil et al. 2012). Existen más de 100 variedades identificadas globalmente de microcistinas que pueden causar la muerte en vertebrados por hemorragia del hígado (Merel et al. 2013, Puddick et al. 2016). La ruta más común de intoxicación humana por microcistinas es la vía oral por ingestión de agua, plantas o animales contaminados, aunque también ocurre por inhalación o por contacto directo con la piel (WHO 2003). Los efectos negativos de las microcistinas pueden presentarse desde algunos minutos hasta varios días después de la exposición a las biotoxinas (Zanchett y Oliveira-Filho, 2013).

Numerosas cianobacterias planctónicas tienen, en general, una tasa de crecimiento mayor que la de muchos autótrofos eucariontes y pueden competir exitosamente cuando la temperatura aumenta (Paerl y Huisman 2009). Tal es el caso de Microcystis, cuyo crecimiento óptimo está regulado, entre otros factores, por la disponibilidad de nitrógeno $(\mathrm{N})$ y fósforo (P) (Harke et al. 2016). Asimismo, su capacidad de almacenar nutrientes le permite crecer en cuerpos de agua desde mesotróficos hasta hipertróficos (Gobler et al. 2016).

Se ha demostrado que, entre los nutrientes implicados en el metabolismo, el $\mathrm{N}$, además de sus funciones como elemento nutritivo esencial, puede modular la toxicidad de Microcystis (Puddick et al. 2016). La limitación de N retrasa la síntesis de microcistinas (Dai et al. 2008). Por otra parte, el P, en concentraciones ambientales reducidas, tiene poco efecto sobre la producción de microcistinas (Dai et al. 2008), y Microcystis tiene la capacidad de usar sulfuros como reemplazo (Puddick et al. 2016).

En la zona urbana de Xochimilco, Ciudad de México, específicamente en las aguas de la Pista Olímpica de Remo y Canotaje Virgilio Uribe, existe un problema ambiental desde hace varias décadas, debido a la presencia y permanencia de florecimientos derivados del crecimiento excesivo de Microcystis (Pineda-Mendoza et al. 2011).

Considerando el potencial nocivo de Microcystis sobre la biota y el ambiente a nivel mundial, los objetivos de interés del presente trabajo de investigación fueron: 1) determinar la influencia de concentraciones crecientes de N y P sobre el crecimiento de Microcystis y 2) evaluar el potencial toxígeno de dos cepas de Microcystis, tomando como organismo experimental al crustáceo Artemia. 
La Pista Olímpica, escogida para realizar este trabajo, es un ejemplo de ecosistema eutrófico, debido a descargas de aguas residuales, donde Microcystis suele desarrollar frecuentemente florecimientos de diversa intensidad (Tavera et al. 2000, Alva-Martínez et al. 2004, 2007a, 2007b, 2009).

\section{MATERIALES Y MÉTODOS}

\section{Recolección y aislamiento de Microcystis}

Microcystis fue recolectada en abril de 2017 en la Pista Olímpica (19 16' 20.58” N, 99 6' 16.72” O) de la Ciudad de México. Este cuerpo de agua mide aproximadamente $2200 \mathrm{~m}$ de largo, $125 \mathrm{~m}$ de ancho, tiene $2 \mathrm{~m}$ de profundidad y mantiene su volumen mediante aporte pluvial y vertido de aguas tratadas (Pineda-Mendoza et al. 2011).

Se recolectaron 51 de agua superficial con botella van Dorn. De este volumen se almacenó $1 \mathrm{~L}$ del agua en un frasco de plástico, colocado y trasportado en una hielera $\left(\sim 8^{\circ} \mathrm{C}\right)$ hasta el laboratorio, previo al aislamiento de Microcystis. La muestra de agua se mantuvo fresca y en la oscuridad por un tiempo $<30$ min antes de llegar al laboratorio. El material se agitó vigorosamente por $1 \mathrm{~min}$ para separar las colonias. Los cultivos clonales se realizaron usando el método de micropipetas y diluciones seriadas bajo el microscopio (Andersen y Kawachi 2005), donde una célula de Microcystis se aisló y multiplicó clonalmente en tubos estériles con medio basal Bold $(0.5 \mathrm{~mL})$. En este estudio se utilizaron dos cepas de Microcystis denominadas Mc17C2 y Mc17C3.

Dichas cepas crecieron en monocultivos in vitro en una cámara iluminada con irradianza de 19.6 $\mu \mathrm{mol} / \mathrm{m}^{2} / \mathrm{s}$, con un fotoperiodo de $12 \mathrm{~h}$ luz: $12 \mathrm{~h}$ oscuridad, a temperatura ambiente $\left(\sim 24 \pm 2{ }^{\circ} \mathrm{C}\right)$. Los cultivos desarrollados de Microcystis se transfirieron y mantuvieron en crecimiento exponencial en un volumen de $250 \mathrm{~mL}$ antes de los experimentos de crecimiento y mortalidad. Cabe destacar que las cepas de Microcystis se distinguieron por su crecimiento unicelular en todos los monocultivos, aunque esporádicamente se observó formación de triadas.

\section{Influencia del nitrógeno y el fósforo sobre el cre- cimiento de Microcystis}

Se utilizó la cepa Mc17C3 para evaluar el efecto de un gradiente de concentración de $\mathrm{N}_{\text {total }}(1.47-29.40 \mathrm{nM})$ y $P_{\text {total }}(0.86-17.21 \mathrm{nM})$ sobre el crecimiento. El medio basal Bold $\left(\mathrm{N}_{\text {total }}=2.94 \mathrm{mM}\right.$ y $\mathrm{P}_{\text {total }}=1.72 \mathrm{mM}$ [Andersen et al. 2005]) se utilizó como referencia estándar en las combinaciones de N y P empleadas para el crecimiento de Microcystis. En este sentido, la combinación de nutrientes asignada a los cultivos experimentales de Microcystis se denominó $1 / 2 \times$, $1 \times, 2 \times, 6 \times$ y $10 \times$ (submúltiplos y múltiplos del valor de $\mathrm{N}$ y $\mathrm{P}$ agregado a los cultivos considerando el contenido del medio basal Bold).

Los experimentos de crecimiento $(1 / 2 \times, 1 \times, 2 \times$, $6 \times$ y $10 \times)$ se realizaron por triplicado en matraces Erlenmeyer (1 L) con un volumen inicial de $700 \mathrm{~mL}$. El crecimiento se evaluó durante 15 días en las condiciones físicas descritas. Los cultivos experimentales iniciaron con abundancias celulares de $10^{4} \mathrm{cel} / \mathrm{mL}$. El crecimiento de Microcystis se cuantificó usando un microscopio compuesto (Zeiss Standard 25) y cámara de Neubauer (Guillard y Sieracki 2005) cada dos días. La tasa de crecimiento se calculó con base en la pendiente de una regresión lineal transformando a logaritmo natural los datos de abundancia de la fase del crecimiento exponencial (Wood et al. 2005).

La concentración de clorofila $a$ en una alícuota de $25 \mathrm{~mL}$ se determinó por espectrofotometría siguiendo el método de extracción pasiva (Jeffrey y Humphrey 1975). Los pigmentos clorofílicos fueron extraídos en la oscuridad durante $24 \mathrm{~h} \mathrm{a} 4{ }^{\circ} \mathrm{C}$, utilizando acetona al $90 \%$.

\section{Bioensayos de toxigenicidad de Microcystis}

La potencial toxigenicidad de Microcystis (cepas Mc17C2 y Mc17C3) se investigó por medio de bioensayos de mortalidad del crustáceo Artemia sp. (Vanhaecke et al. 1981). Artemia sp. se cultivó eclosionando quistes comerciales según el método propuesto por Sorgeloos et al. (1986). Antes del cultivo se filtró $(0.7 \mu \mathrm{m})$ y esterilizó agua de mar con una salinidad de 34 UPS. Los quistes eclosionaron en agua de mar diluida (salinidad 27 UPS) a temperatura de $25^{\circ} \mathrm{C}( \pm 2)$, con un fotoperiodo de $12 \mathrm{~h} \mathrm{luz:} 12 \mathrm{~h}$ oscuridad. En los bioensayos se usaron individuos juveniles de Artemia sp. con la boca y el sistema digestivo desarrollados. Antes de los bioensayos se confirmó la ingestión de células vivas de Microcystis a través de microscopía de epifluorescencia (Fig. 1). Todos los bioensayos se realizaron en la oscuridad para no alterar la abundancia celular de Microcystis, a temperatura de 23 a $26{ }^{\circ} \mathrm{C}$. Como control experimental negativo, Artemia sp. fue alimentada con microalgas no tóxicas de los géneros Scenedesmus y Pseudokirchneriella. El rango de abundancia y la cepa de Microcystis expuesta como alimento a Artemia se describen en el cuadro I. Los controles negativos y las pruebas experimentales de alimentación se realizaron por sextuplicado en todos los casos. Los bioensayos se realizaron en frascos de cristal $(8 \mathrm{~mL})$ 


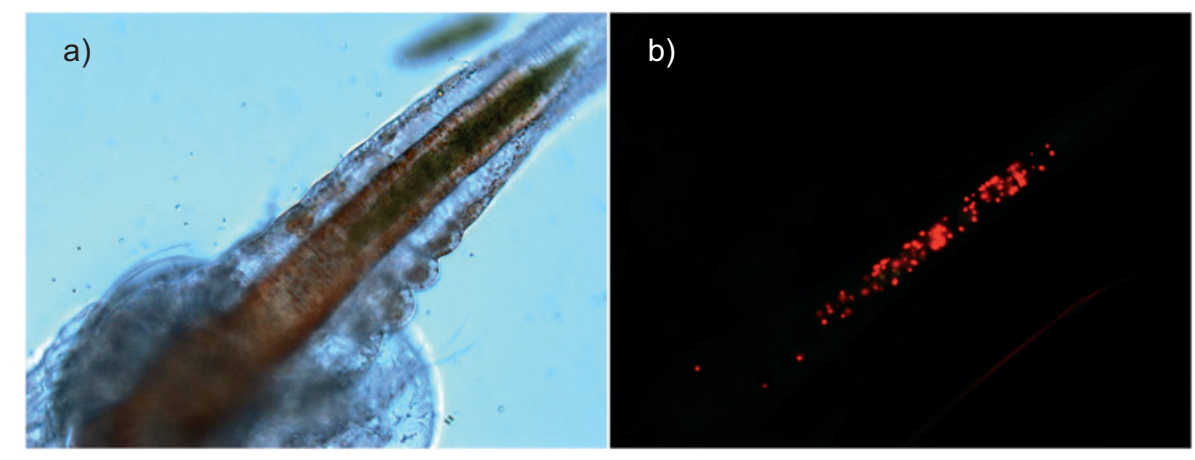

Fig. 1. (a) Vista del tracto digestivo de un juvenil de Artemia sp. observado en campo claro $(100 \times)$. (b) Fluorescencia de la ficoeritrina $(585 \mathrm{~nm})$ en células de Microcystis sp. contenidas en el tracto digestivo de Artemia sp. $(100 \times)$.

CUADRO I. ABUNDANCIA DE Microcystis UTILIZADA EN LOS BIOENSAYOS DE Artemia sp.

\begin{tabular}{ccc}
\hline Bioensayo & Cepa & $\begin{array}{c}\text { Abundancia } \\
\times 10^{3}(\mathrm{cel} / \mathrm{mL})\end{array}$ \\
\hline B1 & Mc17C2 & 5,50 y 300 \\
B2 & Mc17C3 & 21,100 y 1000 \\
B3 & Mc17C3 & 50,500 y 5000 \\
\hline
\end{tabular}

con un volumen de $5 \mathrm{~mL}$ de agua marina diluida (salinidad 27 UPS), donde un organismo de Artemia se alimentó con la abundancia señalada en el cuadro I. El gradiente de abundancia empleado en los bioensayos se realizó por dilución (agua de mar y cultivo de Microcystis), donde se conservó una salinidad de 27 UPS en todos los casos. La mortalidad se cuantificó a las 24, 48 y $72 \mathrm{~h}$ en todos los bioensayos.

Para comparar la toxicidad de Microcystis con resultados de otros estudios, se calculó la equivalencia entre la abundancia celular (cel $/ \mathrm{mL})$ usada en este trabajo y la biomasa seca $(\mathrm{mg} / \mathrm{mL})$ usada por otros autores, considerando que una célula tuvo una equivalencia en peso de $1.21 \times 10^{-11} \mathrm{~g}(\mathrm{Hu} 2014)$.

\section{Análisis de resultados}

Los datos de abundancia, tasa de crecimiento y concentración de clorofila $a$ se promediaron y graficaron con el programa SigmaPlot (v. 13, Systat Sofware, San Jose, CA, EUA). Además, se graficó el porcentaje de mortalidad de Artemia sp. $(\mathrm{n}=4)$ de los controles y de los tratamientos con alimentación a las 24,48 y $72 \mathrm{~h}$. Las tasas de crecimiento determinadas en diferentes concentraciones de nutrimentos $(1 / 2 \times, 1 \times, 2 \times, 6 \times$ y $10 \times)$ se analizaron estadísticamente mediante un análisis de varianza (ANOVA) de una vía (SigmaPlot 13). Previamente se realizaron las pruebas de normalidad (Shapiro-Wilk) y homocedasticidad que confirmaron las condiciones de parametricidad de los datos de crecimiento. El valor de significancia (p) de las pruebas estadísticas fue de 0.05 .

\section{RESULTADOS}

\section{Crecimiento de Microcystis en cultivos enrique- cidos}

Microcystis (cepas Mc17C2 y Mc17C3) fue cultivada y creció satisfactoriamente en condiciones controladas de laboratorio usando los concentrados $1 \times$ a $10 \times$ del medio basal Bold (Fig. 2). Microcystis no creció en el medio Bold diluido $(1 / 2 \times)$ y su crecimiento celular y producción de clorofila $a$ declinaron al iniciar el cultivo (Figs. 2 y 3). El aumento de la concentración de nutrientes disueltos $(1 \times$ a $10 \times)$ produjo un incremento en las tasas de crecimiento de Microcystis (Fig. 4), pero el ANOVA $(\mathrm{n}=3)$ no mostró diferencias significativas en las tasas de crecimiento de los experimentos $1 \times\left(0.49 \pm 0.10 \mathrm{~d}^{-1}\right), 6 \times(0.58$ $\left.\pm 0.12 \mathrm{~d}^{-1}\right)$ y $10 \times\left(0.50 \pm 0.05 \mathrm{~d}^{-1}\right)(\mathrm{p}=0.05$, Fig. 4) Sin embargo, el crecimiento óptimo de Microcystis ocurrió en los cultivos $6 \times\left(0.58 \mathrm{~d}^{-1}\right)$. En el medio Bold $2 \times$, a pesar de la concentración de nutrientes, Microcystis redujo su tasa máxima de crecimiento $\left(0.33 \mathrm{~d}^{-1}\right)$, pero no mostró diferencias estadísticamente significativas respecto a las tasas determinadas en los cultivos $1 \times$ y $10 \times$, con excepción del medio Bold $6 \times($ ANOVA, p $=0.05$, Fig. 4). Independientemente de la concentración de nutrientes (medio Bold $1 \times$ a $10 \times$ ), la fase del crecimiento exponencial de Microcystis tuvo una duración < siete días para todos los tratamientos, y las abundancias máximas fluctuaron entre $8.83 \times 10^{4}$ y $6.52 \times 10^{5} \mathrm{cel} / \mathrm{ml}$ (Fig. 2). 


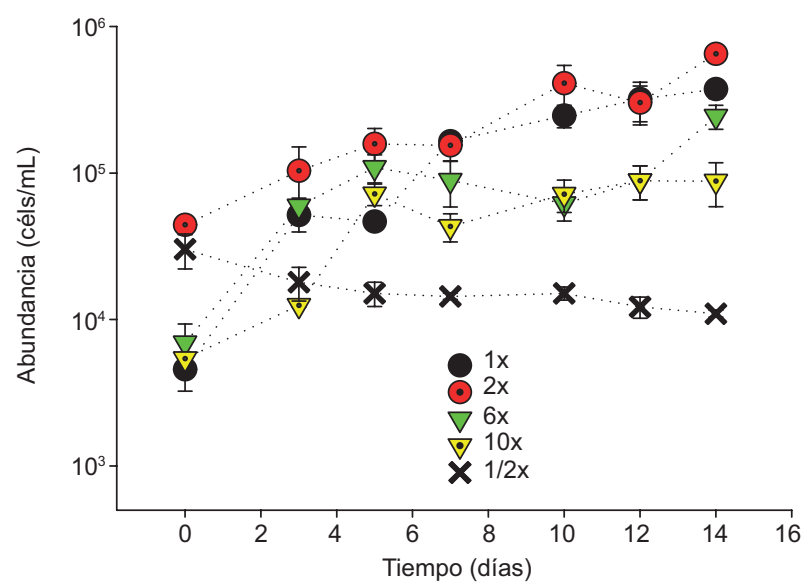

Fig. 2. Crecimiento de Microcystis (cepa Mc17C3) determinado en diferentes concentraciones de nutrientes. Cada punto indica el promedio del crecimiento de Microcystis en el tiempo $(\mathrm{n}=3)$. Las barras verticales indican la desviación estándar $(n=3)$.

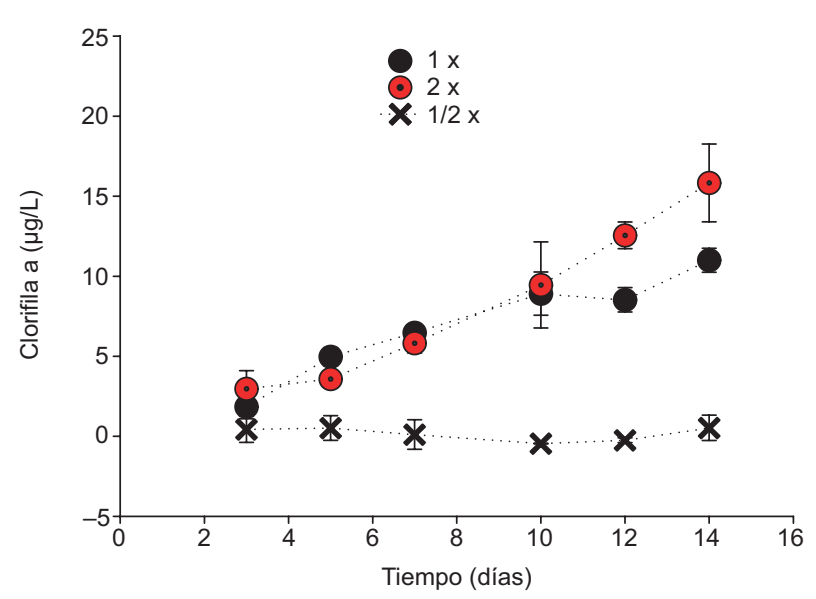

Fig. 3. Concentración de clorofila $a$ obtenida en cultivos monoclonales de Microcystis (cepa Mc17C3) en diferentes concentraciones de nutrientes. Los símbolos representan los promedios y las barras verticales indican la desviación estándar.

La producción de clorofila $a$ durante el crecimiento de Microcystis no mostró diferencias significativas en los medios $1 \times$ y $2 \times$, donde el máximo registrado fue de $11.0 \mu \mathrm{g} \mathrm{cl} a / \mathrm{L} \mathrm{y} 15.8 \mu \mathrm{g} \mathrm{cl} a / \mathrm{L}$ (Fig. 3), respectivamente.

\section{Mortalidad por ingestión de Microcystis}

Artemia sp. se alimentó de distintas cantidades de Microcystis (Mc17C2 y Mc17C3) en los bioensayos, confirmándose que a mayor abundancia de Microcystis aumenta la tasa de mortalidad de Artemia (Fig. 5). En general, Artemia sp. no fue sensible a la ingestión

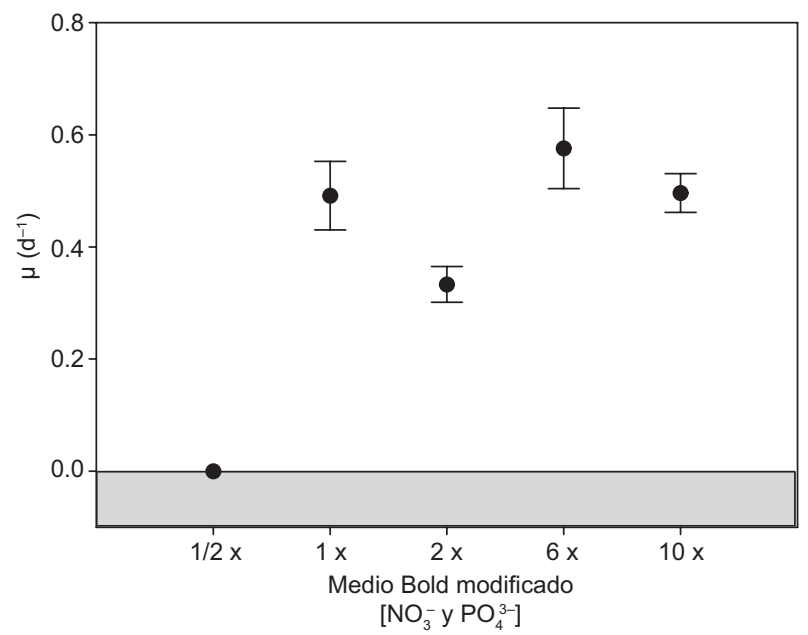

Fig. 4. Tasa de crecimiento ( $\mu$ ) de Microcystis (cepa Mc17C3) obtenida en diferentes concentrados del medio de cultivo Bold. Las barras verticales indican la desviación estándar $(n=3)$.

de Microcystis en las primeras $24 \mathrm{~h}$, excepto en el bioensayo B1 con abundancia de $3 \times 10^{5} \mathrm{cel} / \mathrm{ml}$ de la cepa Mc17C2 (Fig. 5). La tasa de mortalidad de juveniles de Artemia, producto de una intoxicación potencial, se agudizó a las 48 y $72 \mathrm{~h}$ (bioensayos B1 y B3), llegando a producir $>50 \%$ de decesos cuando la abundancia fluctuó de $3 \times 10^{5}$ a $3 \times 10^{6} \mathrm{cel} / \mathrm{ml}$ (Fig. 5). Cabe destacar que en el bioensayo B2 se obtuvo la máxima tasa de mortalidad (100\%) de Artemia a las $72 \mathrm{~h}$ de haber sido alimentada con $1 \times 10^{5}$ y $3 \times 10^{6} \mathrm{cel} /$ $\mathrm{ml}$ de la cepa Mc17C3. Sin embargo, en el bioensayo B3, a pesar de que se utilizó una mayor abundancia de Microcystis ( $\geq 5$ veces) que en el bioensayo B2, únicamente se alcanzó un máximo de mortalidad de 66 $\%$ al término de $72 \mathrm{~h}$ (Fig. 5). La valoración del efecto máximo de mortalidad indica que la cepa $\mathrm{Mc} 17 \mathrm{C} 3$ de Microcystis fue potencialmente más letal que la cepa Mc17C2 (Fig. 5). La exposición de Artemia sp. a abundancia $\leq 10^{4} \mathrm{cel} / \mathrm{ml}$ de Microcystis causó mortalidad inferior al $50 \%$ en la mayoría de los bioensayos.

\section{DISCUSIÓN}

Frecuentemente los florecimientos nocivos de cianobacterias como Microcystis provocan múltiples problemas ambientales, socioeconómicos y de salud en ecosistemas acuáticos epicontinentales a nivel global (Harke et al. 2016). En la zona urbana de Xochimilco, específicamente en las aguas de la Pista Olímpica, existe un problema ambiental desde hace décadas debido a la presencia y permanencia de un 

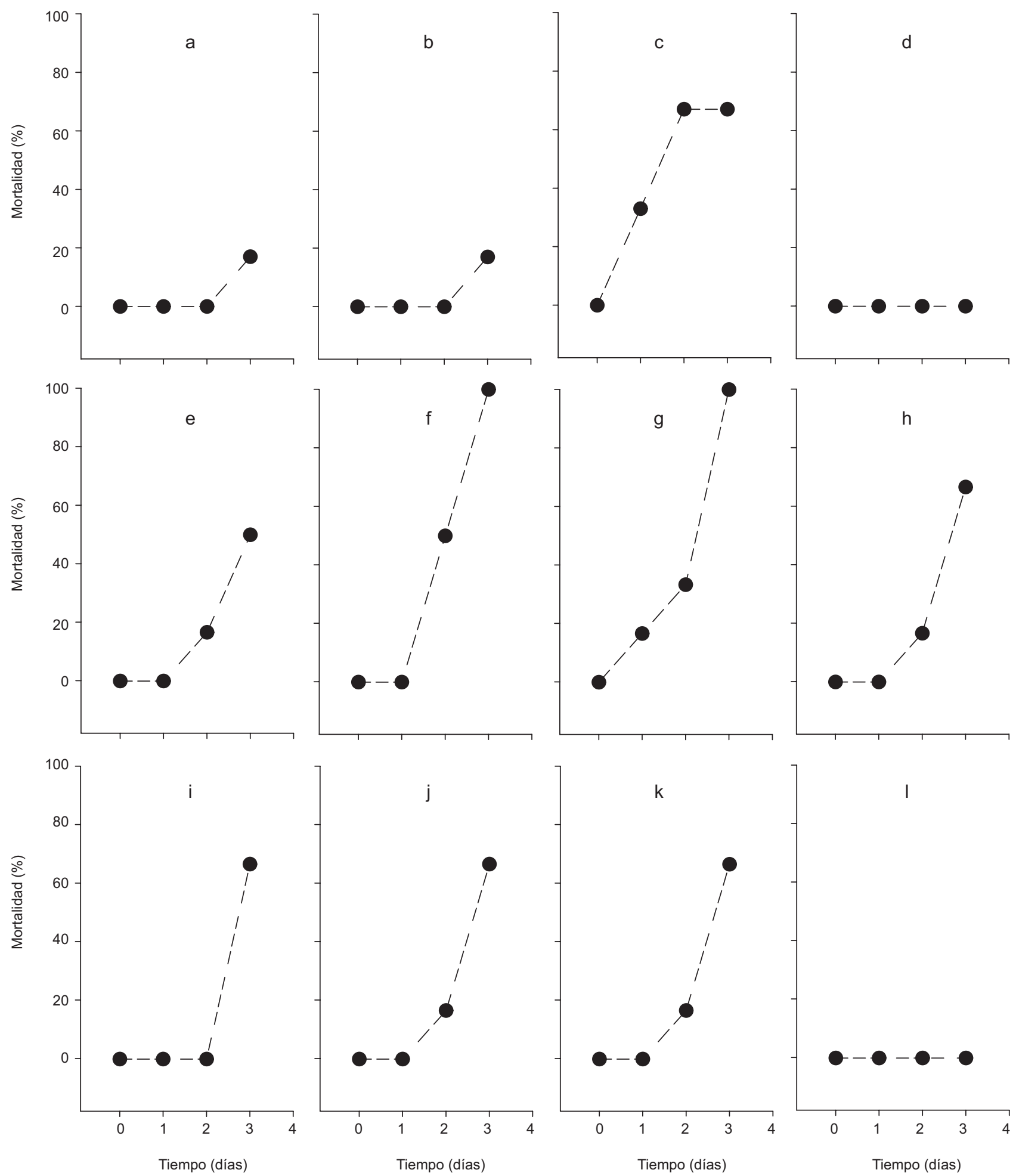

Fig. 5. Tasa de mortalidad de Artemia sp. por exposición e ingestión de Microcystis a diferentes concentraciones. (a-c) Bioensayo 1 $\left(5,50\right.$ y $300 \times 10^{3} \mathrm{cel} / \mathrm{mL}$, respectivamente). (d) Control. (e-g) Bioensayo $2\left(21,100\right.$ y $1000 \times 10^{3}$ cel $/ \mathrm{mL}$, respectivamente). (h) Control. (i-k) Bioensayo 3 (50, 500 y $5000 \times 10^{3} \mathrm{cel} / \mathrm{ml}, \mathrm{i}-\mathrm{k}$, respectivamente). (1) Control. Cada punto indica el promedio porcentual de mortalidad en el tiempo $(\mathrm{n}=4)$. En todos los controles Artemia se alimentó con fitoplancton no tóxico. 
florecimiento derivado del crecimiento excesivo de Microcystis (Pineda-Mendoza et al. 2011). En esta región, los FAN de cianobacterias no han recibido la suficiente atención y, en general, se ignoran los riesgos que representan para la salud humana y la biota (Pérez-Morales et al. 2016).

Numerosos cuerpos de agua de Xochimilco están eutrofizados por exceso de nutrientes. La Pista Olímpica, por ejemplo, recibe aguas tratadas del Cerro de la Estrella desde hace varias décadas (Arcos-Ramos et al. 2012). El efecto de la carga de nutrientes en el agua no sólo ha favorecido la permanencia de los FAN de Microcystis, sino que también podría ser factor promotor de la producción y acumulación masiva de toxinas dañinas para la biota, incluyendo el hombre. Por ello, es indispensable comprender los impactos de la eutrofización por altas concentraciones de N y P sobre el crecimiento de Microcystis, así como advertir la potencial toxigenicidad de esta cianobacteria en el ambiente.

\section{Nutrientes y crecimiento de Microcystis}

En este trabajo, la tasa óptima de crecimiento de Microcystis en condiciones de cultivo se asoció con concentraciones elevadas de $\mathrm{N}$ y de $\mathrm{P}$ (medio Bold 6 $\times$ ). Estos resultados coinciden con los realizados en distintas regiones, como en el caso de una cepa aislada de una laguna de Filipinas (Baldia et al. 2007). En ese mismo sentido, Vézie et al. (2002) demostraron que una proporción alta de N:P favorece el crecimiento de Microcystis, siempre y cuando la concentración de $\mathrm{N}$ supere un nivel mínimo $(0.05 \mathrm{mg} / \mathrm{L})$, tomando en cuenta la incapacidad de esta cianobacteria para fijar $\mathrm{N}$ atmosférico. Es importante destacar que los nutrientes influyen sobre la abundancia tanto de cepas tóxicas como no tóxicas; estas últimas los requieren en menor concentración para alcanzar la tasa máxima de crecimiento, en tanto que las cepas tóxicas los necesitan en mayores concentraciones (Davis et al. 2009, Burford et al. 2018). La ausencia de crecimiento de Microcystis en el experimento con menor concentración de nutrientes podría relacionarse con el aumento o mayor influencia en la síntesis de compuestos de reserva a expensas de la disminución en la división celular (Cordeiro et al. 2017).

Adicionalmente, la respuesta interespecífica de crecimiento de Microcystis en diferentes concentraciones de $\mathrm{N}$ y $\mathrm{P}$, ha mostrado resultados muy variables (Parrish 2014); por lo anterior, diversos autores coinciden en la dificultad de definir los límites de $\mathrm{N} \mathrm{y}$ P que promueven el desarrollo y la formación súbita de sus florecimientos. En consecuencia, es importante estudiar el crecimiento de diferentes cepas del género, ya que las respuestas génicas y fisiológicas de poblaciones de distintas localidades y ambientes pueden diferir significativamente (Vézie et al. 2002).

Las tasas máximas de crecimiento de Microcystis obtenidas en este estudio $\left(0.58\right.$ y $0.50 \mathrm{~d}^{-1}$, medios Bold $6 \times$ y $10 \times$ ) son superiores a las documentadas en otros reportes que usaron el mismo medio Bold. La tasa óptima de crecimiento, obtenida usando el medio Bold $6 \times$, permite inferir que una probable eutrofización del ambiente favorecería el mantenimiento y crecimiento de la cianobacteria. En la condición opuesta, en cuanto a limitación de $\mathrm{N}$ y de $\mathrm{P}$ en cultivo, se ubican tasas de crecimiento menores (0.15-0.18 d $\mathrm{d}^{-1}$ [Imai et al. 2009, Che et al. 2017]), como ocurrió en este caso con el crecimiento en el medio con menor concentración de nutrientes. Por otra parte, también se ha señalado que Microcystis puede adaptarse y crecer eficientemente en condiciones reducidas de $\mathrm{N}$, pero no en ausencia de $\mathrm{P}$ (Wu et al. 2015). Cabe agregar que la tasa de asimilación de diferentes concentraciones de $\mathrm{P}$ varía durante las etapas del crecimiento de Microcystis; puede aumentar en los primeros días de cultivo y luego disminuir (Ghaffar et al. 2017), como en los experimentos $1 \times$ y $2 \times$ aquí presentados. Algunos autores consideran que el $\mathrm{P}$ es el principal factor limitante en la formación y permanencia de los FAN de Microcystis en aguas epicontinentales (Harke et al. 2012).

\section{Toxigenicidad de Microcystis en los consumidores}

Las toxinas producidas por cianobacterias afectan a organismos acuáticos a distintos niveles. En este trabajo, las cepas Mc17C2 y Mc17C3 provocaron inmovilidad, letargo y mortalidad de juveniles de Artemia, como ocurrió en estudios previos (Arzate-Cárdenas et al. 2010, Vasconcelos et al. 2010, entre otros). En ese sentido, las toxinas producidas por Microcystis podrían considerarse un mecanismo de defensa contra organismos fitoplanctívoros y, al mismo tiempo, afectarían las tasas de alimentación, reproducción y supervivencia de algunos depredadores (Holland y Kinnear 2013, Zanchett y Oliveira-Filho 2013).

La mortalidad de Artemia en los bioensayos estuvo relacionada directamente con el aumento de la abundancia celular de Microcystis, ya que se ha encontrado que al aumentar la abundancia de este microorganismo disminuye la supervivencia de los crustáceos Artemia y Daphnia magna (Arzate-Cárdenas et al. 2010). Cabe señalar que la biomasa seca de Microcystis se ha utilizado en varios bioensayos de toxicidad (Cuadro II), en los cuales la mortalidad de Artemia sp. ha sido similar a la de este estudio, hecho que revela la utilidad de su potencial nocivo como primer diagnóstico. 
CUADRO II. ESTUDIOS DOCUMENTADOS SOBRE MORTALIDAD DE Artemia POR INGESTIÓN DE Microcystis.

\begin{tabular}{|c|c|c|c|c|c|c|c|}
\hline Especie & Organismo & $\begin{array}{c}\text { Abundancia } \\
\times 10^{3} \\
(\mathrm{cel} / \mathrm{mL})\end{array}$ & $\begin{array}{c}\text { Conversión } \\
\text { Peso-célula } \\
\text { (cel/mL) } \\
\text { (Hu 2014) }\end{array}$ & $\begin{array}{l}\text { Compuesto nocivo } \\
\text { aplicado }\end{array}$ & $\begin{array}{l}\text { Compuesto } \\
\text { tóxico }\end{array}$ & $\begin{array}{l}\text { Mortalida } \\
(\%)\end{array}$ & Referencia \\
\hline Microcystis sp. & Artemia salina & $\mathrm{Nd}$ & $8.26 \times 10^{8}$ & $\begin{array}{l}10 \mathrm{mg} / \mathrm{mL} \text { biomasa } \\
\text { liofilizada }(24 \mathrm{~h})\end{array}$ & MC-LR & $>50$ & Douma et al. 2017 \\
\hline $\begin{array}{l}\text { Microcystis } \\
\text { aeruginosa }\end{array}$ & $\begin{array}{c}\text { Artemia } \\
\text { franciscana }\end{array}$ & 1500 & - & $3.62 \mu \mathrm{M}(24 \mathrm{~h})$ & MC-LR & $>80$ & D'ors et al. 2013 \\
\hline Microcystis sp. & Artemia salina & $\mathrm{Nd}$ & $4.13 \times 10^{9}$ & $\begin{array}{c}50 \mathrm{mg} / \mathrm{mL} \text { biomasa } \\
\text { seca }(48 \mathrm{~h})\end{array}$ & MC-LR & $>50$ & Metcalf et al. 2002 \\
\hline $\begin{array}{l}\text { Microcystis } \\
\text { ichthyoblade }\end{array}$ & Artemia salina & $\mathrm{Nd}$ & $3.30 \times 10^{9}$ & $\begin{array}{c}40 \mathrm{mg} / \mathrm{mL} \text { biomasa } \\
\text { seca }(24 \mathrm{~h})\end{array}$ & MC-LR & 40 & Sabour et al. 2002 \\
\hline Microcystis sp. & Artemia salina & $\mathrm{Nd}$ & $\begin{array}{c}5.78 \times 10^{7} \\
-6.52 \times 10^{8}\end{array}$ & $\begin{array}{c}0.7-7.9 \mathrm{mg} / \mathrm{mL} \\
\text { biomasa seca }(24 \mathrm{~h})\end{array}$ & MC-LR & $>50$ & $\begin{array}{l}\text { Kyselková y } \\
\text { Maršálek } 2000\end{array}$ \\
\hline Microcystis sp. & Artemia salina & $\mathrm{Nd}$ & $8.26 \times 10^{8}$ & $\begin{array}{c}10 \mathrm{mg} / \mathrm{mL} \text { biomasa } \\
\text { seca }(48 \mathrm{~h})\end{array}$ & MC-LR & $>65$ & Vézie et al. 1996 \\
\hline $\begin{array}{l}\text { Microcystis } \\
\text { aeruginosa }\end{array}$ & Artemia salina & $\mathrm{Nd}$ & $\begin{array}{c}4.13 \times 10^{8} \\
-7.02 \times 10^{8}\end{array}$ & $\begin{array}{c}5.0-8.5 \mathrm{mg} / \mathrm{mL} \\
\text { biomasa seca }(24 \mathrm{~h})\end{array}$ & $\mathrm{Nd}$ & $>80$ & $\begin{array}{l}\text { Kiviranta et al. } \\
1991\end{array}$ \\
\hline $\begin{array}{l}\text { Microcystis } \\
(\mathrm{Mc} 17 \mathrm{C} 2)\end{array}$ & Artemia sp. & 300 & - & $\mathrm{Nd}$ & $\mathrm{Nd}$ & $>50$ & Este estudio \\
\hline $\begin{array}{l}\text { Microcystis } \\
(\mathrm{Mc} 17 \mathrm{C} 3)\end{array}$ & Artemia sp. & $\begin{array}{c}21,50,100 \\
500,1000 \mathrm{y} \\
5000\end{array}$ & - & $\mathrm{Nd}$ & $\mathrm{Nd}$ & $>50$ & Este estudio \\
\hline
\end{tabular}

MC-LR: microcistina LR, Nd: no disponible.

En el cuadro II se aprecia que hubo mortalidad significativa ( $>50 \%$ ) de organismos de Artemia alimentados con cultivos de Microcystis mucho menos abundantes que los usados en otros estudios. Este resultado permite inferir la potencial toxigenicidad de las cepas Mc17C2 y Mc17C3, ya que existe relación entre el aumento de la mortalidad de Artemia y el incremento proporcional en la concentración de hepatotoxinas y neurotoxinas (Kiviranta et al. 1991, Campbell et al. 1994).

La diferencia en la mortalidad de Artemia producida por las cepas Mc17C2 y Mc17C3 (67-100\% vs. 50-67\%, respectivamente) podría atribuirse a una mayor concentración de compuestos nocivos. D'ors et al. (2013) señalan diferencias en la producción de compuestos nocivos entre distintas cepas que tienen efectos diferentes sobre los bioensayos. La mortalidad de Artemia estaría asociada al efecto de altas concentraciones de compuestos nocivos que inhiben la producción de enzimas digestivas en crustáceos, indispensables para desintegrar y digerir la biomasa fitoplanctónica de sus presas (Agrawal et al. 2005).

\section{CONCLUSIONES}

El incremento y la disponibilidad de nutrientes disueltos ( $\mathrm{N}$ y $\mathrm{P}$ ) en cultivos promueven mayores tasas de crecimiento de poblaciones de Microcystis. Por tanto, es posible que la descarga de aguas tratadas, ricas en nutrientes, al ambiente acuático de la Pista Olímpica de Remo y Canotaje Virgilio Uribe continúe teniendo implicaciones ecológicas en el mantenimiento, el crecimiento y la potencial producción de toxinas de Microcystis en el ambiente. Aunque el presente estudio permitió estimar que Microcystis posee un mecanismo de toxigenicidad potencialmente nocivo para la biota acuática, no se identificaron los metabolitos que pudieron causar mortalidad en juveniles de Artemia. En consecuencia, se requiere profundizar en el conocimiento y comprensión del funcionamiento de la ecología química de poblaciones toxígenas de Microcystis en éste y otros ambientes acuáticos epicontinentales. 


\section{REFERENCIAS}

Agrawal M.K., Bagchi D. y Bagchi S.N. (2005). Cysteine and serine protease-mediated proteolysis in body homogenate of a zooplankter, Moina macropa, is inhibited by the toxic cyanobacterium Microcystis aeruginosa PCC7806. Comp. Biochem. Physiol. 141, 33-41. https://doi.org/10.1016/j.cbpc.2005.01.002

Alva-Martínez A.F., Sarma S.S.S. y Nandini S. (2004). Population growth of Daphnia pulex (Cladocera) on a mixed diet (Microcystis aeruginosa with Chlorella or Scenedesmus). Crustaceana 77, 973-988. https://doi. org/10.1163/1568540042781720

Alva-Martínez A.F., Sarma S.S.S. y Nandini. S. (2007a). Effect of mixed diets (cyanobacteria and green algae) on the population growth of the cladocerans Ceriodaphnia dubia and Moina macrocopa. Aquat. Ecol. 41, 579-585. https://doi.org/10.1007/s10452-007-9115-1

Alva-Martínez A.F., Sarma S.S.S. y Nandini S. (2007b). Population dynamics of Brachionus calyciflorus and Brachionus havanaensis (Rotifera) on mixed diets with Microcystis aeruginosa and green algae. Hidrobiológica 17, 59-67.

Alva-Martínez A.F., Fernández, R., Sarma S.S.S. y Nandini S. (2009). Effect of mixed toxic diets (Microcystis and Chlorella) on the rotifers Brachionus calyciflorus and Brachionus havanaensis cultured alone and together. Limnologica 39, 302-305. https://doi.org/10.1016/j. limno.2009.06.002

Andersen R.A., Berges J.A., Harrison P.J. y Watanabe M.M. (2005). Appendix A-recipes for freshwater and seawater media. En: Algal culturing techniques (Andersen R.A., Ed.). Elsevier Academic Press, London, Reino Unido, 429-538.

Andersen R.A. y Kawashi M. (2005). Traditional microalgae isolation techniques. En: Algal culturing techniques (Andersen R.A., Ed.). Elsevier Academic Press, London, Reino Unido, 83-100.

Arcos-Ramos R., Cruz-Martínez P. y Díaz-Hernández I.S. (2012). Estimación de la salinidad del lago de Xochimilco considerando ciertos parámetros químicos (aniones y cationes). En: Contribuciones al conocimiento de la ecotoxicología y química ambiental en México (Pica-Granados Y. y Ramírez-Romero P., Eds.). Instituto Mexicano de Tecnología del Agua, Morelos, México, 410-420

Arzate-Cárdenas M.A., Olvera-Ramírez R. y MartínezJerónimo F. (2010). Microcystis toxigenic strains in urban lakes: a case of study in Mexico City. Ecotoxicology 19, 1157-1165. https://doi.org/10.1007/ s10646-010-0499-7

Baldia S.F., Evangelista A.D., Aralar E.V. y Santiago A.E. (2007). Nitrogen and phosphorus utilization in the cyanobacterium Microcystis aeruginosa isolated from Laguna de Bay, Philippines. J. Appl. Phycol. 19, 607-613. https://doi.org/10.1007/s10811-007-9209-0

Burford M.A., Hamilton D.P. y Wood S.A. (2018). Emerging $\mathrm{HAB}$ research issues in freshwater environments. En: Global ecology and oceanography of harmful algal blooms (Glibert P.M., Berdalet E., Burford M.A., Pitcher G.C. y Zhou M., Eds.). Springer International Publishing, Cham, Suiza, 381-402. https://doi. org/10.1007/978-3-319-70069-4_20

Campbell D.L., Lawton, L.A., Beattie K.A. y Codd G.A. (1994). Comparative assessment of the specificity of the brine shrimp and microtox assays to hepatotoxic (Microcystin-LR-containing) cyanobacteria. Environ. Toxic. Water 9, 71-77. https://doi.org/10.1002/ tox.2530090109

Che F., Du M. y Yan C. (2017). Arsenate biotransformation by Microcystis aeruginosa under different nitrogen and phosphorus levels. J. Environ. Sci. 66, 41-49. https:// doi.org/10.1016/j.jes.2017.05.041

Cordeiro R.S., Vaz I.C.D., Magalhães S.M.S. y Barbosa F.A.R. (2017). Effects of nutritional conditions on lipid production by cyanobacteria. An. Acad. Bras. Cienc. 89 (3), 2021-2031. https://doi.org/10.1590/00013765201720150707

Dai R., Liu H., Qu J., Zhao X., Ru J. y Hou Y. (2008). Relationship of energy charge and toxin content of Microcystis aeruginosa in nitrogen-limited or phosphorous-limited cultures. Toxicon 51 (4), 649-658. https://doi.org/10.1016/j.toxicon.2007.11.021

Davis T.W., Berry D.L., Boyer G.L. y Glober C.J. (2009). The effects of temperature and nutrients on the growth and dynamics of toxic and non-toxic strains of Microcystis during cyanobacteria blooms. Harmful Algae 8, 715-725. https://doi.org/10.1016/j.hal.2009.02.004

D’ors, A., Bartolomé M.C. y Sánchez-Fortún S. (2013). Toxic risk associated with sporadic occurrences of Microcystis aeruginosa blooms from tidal rivers in marine and estuarine ecosystems and its impact on Artemia franciscana nauplii populations. Chemosphere 90, 2187-2192. https://doi.org/10.1016/j.chemosphere.2012.11.029

Douma, M., Manaut, N., Saqrane, S., El Khalloufi, F., Oudra B. y Loudiki M. (2017). Toxicity assessment and detection of cyanobacterial toxins (microcystins) in a Mediterranean natural lake (Dayete Aoua, Morocco). J. Mater. Environ. Sci. 8 (9), 3247-3251.

García-Mendoza E., Quijano-Scheggia S.I., Olivos-Ortiz A., Núñez-Vázquez E.J. y Pérez-Morales A. (2016). Introducción general. En: Florecimientos algales nocivos en México (García-Mendoza E., Quijano-Scheggia S.I., Olivos-Ortiz A. y Núñez-Vázquez E.J., Eds.). CICESE, Ensenada, México, 10-19. 
Ghaffar S., Stevenson R.J. y Khan Z. (2017). Effect of phosphorus stress on Microcystis aeruginosa growth and phosphorus uptake. Plos One 12 (3), 1-17. https:// doi.org/10.1371/journal.pone.0174349

Gobler C.J., Burkholder J.M., Davis T.W., Harke M.J., Johengen T., Stow C.A. y van de Waal D.B. (2016). The dual role of nitrogen supply in controlling the growth and toxicity of cyanobacteria blooms. Harmful Algae 54, 87-97. https://doi.org/10.1016/j.hal.2016.01.010

Guillard R.R.L. y Sieracki M.S. (2005). Counting cells in cultures with the light microscope. En: Algal culturing techniques (Andersen R.A., Ed.). Elsevier Academic Press, Londres, Reino Unido, 239-252.

Harke M.J., Berry D.L., Ammerman J.W. y Gobler C.J. (2012). Molecular response of the bloom-forming cyanobacterium, Microcystis aeruginosa, to phosphorus limitation. Microb. Ecol. 63 (1), 188-198. https://doi. org/10.1007/s00248-011-9894-8

Harke M.J., Steffen M.M., Gobler C.J., Otten T.G., Wilhelm S.W., Wood S.A. y Paerl H.W. (2016). A review of the global ecology, genomics, and biogeography of the toxic cyanobacterium Microcystis spp. Harmful Algae 54, 4-20. https://doi.org/10.1016/j.hal.2015.12.007

Holland A. y Kinnear S. (2013). Interpreting the possible ecological role(s) of cyanotoxins: Compounds for competitive advantage and/or physiological aide. Mar. Drugs 11, 2239-2258. https://doi.org/10.3390/ md11072239

$\mathrm{Hu}$ W. (2014). Dry weight and cell density of individual algal and cyanobacterial cells for algae. M.Sc. thesis. Faculty of the Graduate School, University of Missouri-Columbia, Columbia, USA, 68 pp.

Imai H., Chang K.H. y Nakano S. (2009). Growth responses of harmful algal species Microcystis (Cyanophyceae) under various environmental conditions. En: Interdisciplinary studies on environmental chemistry. Environmental research in Asia (Obayashi Y., Isobe T., Subramanian A., Suzuki S. y Tanabe S., Eds.) TERRAPUB, Tokio, 269-275.

Jeffrey S.W. y Humphrey G.F. (1975). New spectrophotometric equations for determining chlorophylls $a, b, c$ and $c 2$ in higher plants, algae and natural phytoplankton. Biochem. Physiol. Pflanz.167, 191-194. https:// doi.org/10.1016/s0015-3796(17)30778-3

Kyselková I. y Maršalek B. (2000). Use of Daphnia pulex, Artemia salina and Tubifex for cyanobacterial microcystins toxicity detection. Biologia 55, 637-643.

Kiviranta J., Sivonen K. y Niemelá S.I. (1991). Detection of toxicity of cyanobacteria by Artemia salina bioassay. Environ. Toxic. Water 6, 756-436. https://doi. org/10.1002/tox.2530060407

Merel S., Walker D., Chicana R., Snyder S., Baurès E. y Thomas O. (2013). State of knowledge and concerns on cyanobacterial blooms and cyanotoxins. Environ. Int. 59, 303-327. https://doi.org/10.1016/j. envint.2013.06.013

Metcalf J.S., Lindsay J., Beattie K.A., Birmingham S., Saker M.L., Törökné A.K. y Codd G.A. (2002). Toxicity of cylindrospermopsin to the brine shrimp Artemia salina: Comparisons with protein synthesis inhibitors and microcystins. Toxicon 40, 1115-1120. https://doi. org/10.1016/S0041-0101(02)00105-8

O’Neil J.M., Davis T.W., Burford M.A. y Gobler C.J. (2012). The rise of harmful cyanobacteria blooms: The potential roles of eutrophication and climate change. Harmful Algae 14, 313-334. https://doi.org/10.1016/j. hal.2011.10.027

Paerl H.W. y Huisman J. (2008). Climate. Blooms like it hot. Science 320, 57-58. https://doi.org/10.1126/ science. 1155398

Paerl H.W. y Huisman J. (2009). Climate change: A catalyst for global expansion of harmful cyanobacterial blooms. Env. Microbiol. Rep. 1, 27-37. https://doi. org/10.1111/j.1758-2229.2008.00004.x

Parrish J. (2014). The role of nitrogen and phosphorus in the growth, toxicity, and distribution of the toxic cyanobacteria Microcystis aeruginosa. Master's project and capstones. University of San Francisco, California, USA, $61 \mathrm{pp}$.

Pérez-Morales A., Olivos-Ortiz A., Quijano-Scheggia S.I., Espinosa-Rodríguez C.A. y Jiménez-Santos M.A. (2016). Estado actual del estudio de cianobacterias dulceacuícolas formadoras de florecimientos en el centro de México. En: Florecimientos algales nocivos en México (García-Mendoza E., Quijano-Scheggia S.I., Olivos-Ortiz A. y Núñez-Vázquez E.J., Eds.). CICESE, Ensenada, México, 408-421.

Pineda-Mendoza R.M., Martínez-Jerónimo F., GarduñoSolórzano G. y Olvera-Ramírez R. (2011). Caracterización morfológica y molecular de cianobacterias filamentosas aisladas de florecimientos de tres lagos urbanos eutróficos de la Ciudad de México. Polibotánica 31 (3), 31-50.

Puddick J., Prinsep M.R., Wood S.A., Cary S.C. y Hamilton D.P. (2016). Modulation of microcystin congener abundance following nitrogen depletion of a Microcystis batch culture. Aquat. Ecol. 50, 235-246. https:// doi.org/10.1007/s10452-016-9571-6

Sabour B., Loudiki M., Oudra B., Vasconcelos V., Martins R., Oubraim S. y Fawzi B. (2002). Toxicology of a Microcystis ichthyoblabe waterbloom from Lake Oued Mellah (Morocco). Environ. Toxicol. 17, 24-31. https:// doi.org/10.1002/tox.10028

Sorgeloos P., Lavens P., Lè P., Tackaert W. y Versichele D. (1986). Producción de nauplios de Artemia y quistes decapsulados para usarlos como alimento en centros 
de puesta de peces y crustáceos. En: Manual para el cultivo y uso de Artemia en acuicultura (Sorgeloos P., Lavens P., Lè P., Tackaert W. y Versichele D., Eds.). FAO. Gent, Bélgica, 74-112.

Tavera R., Novelo E. y Comas A. (2000). Chlorococcalean algae (s.l.) from the ecological park of Xochimilco, Mexico. Algological Studies 100, 65-94. https://doi. org/10.1127/algol_stud/100/2000/65

Vanhaecke P., Persoone G., Claus C. y Sorgeloos P. (1981). Proposal for a short-term toxicity test with Artemia nauplii. Ecotox. Environ. Safe. 5, 382-387. https://doi. org/10.1016/0147-6513(81)90012-9

Vasconcelos V., Martins A., Vale M., Antunes A., Azevedo J., Welker M., López O. y Montejano G. (2010). First report and the occurrence of microcystins in planktonic cyanobacteria from Central Mexico. Toxicon 56, 425-431. https://doi.org/10.1016/j.toxicon.2010.04.011

Vézie C., Benoufella F., Sivonen K., Bertru G. y Laplanche A. (1996). Detection of toxicity of cyanobacterial strains using Artemia salina and Microtox ${ }^{\circledR}$ assays compared with mouse bioassay results. Phycologia 35, 198-202. https://doi.org/10.2216/i0031-8884-356s-198.1
Vézie C., Rapala J., Vaitomaa J., Seitsonen J. y Sivonen K. (2002). Effect of nitrogen and phosphorus on growth of toxic and nontoxic Microcystis strains and on intracellular microcystin concentrations. Microb. Ecol. 43 (4), 443-454. https://doi.org/10.1007/s00248-001-0041-9

WHO (2003). Algae and cyanobacteria in fresh water. En: Guidelines for safe recreational water environments. Vol. 1: Coastal and fresh waters. World Health Organization, Geneva, Switzerland, 136-158.

Winder M. y Sommer U. (2012). Phytoplankton response to a changing climate. Hydrobiologia $698,5-16$. https:// doi.org/10.1007/s10750-012-1149-2

Wood A.M., Everroad R.C. y Wingard L.M. (2005). Measuring growth rates in microalgal cultures. En: Algal culturing techniques (Andersen R.A., Ed.). Elsevier Academic Press, Londres, Reino Unido, 269-326.

Wu J., Liu C., Jia R. y Li B. (2015). The correlation between nitrogen phosphorus nutrients and the growth of Microcystis aeruginosa. Adv. Mater. Res. 1065-1069, 3110-3113. https://doi.org/10.4028/www.scientific.net/ amr.1065-1069.3110

Zanchett G. y Oliveira-Filho E.C. (2013). Cyanobacteria and cyanotoxins: From impacts on aquatic ecosystems and human health to anticarcinogenic effects. Toxins 5, 1896-1917. https://doi.org/10.3390/toxins5101896 